\title{
Computational multi-component modelling of electron transport and ohmic heating inside a micro arc-jet thruster
}

\author{
R. Groll \& J. E. Gomez \\ Center of Applied Space Technology and Microgravity, \\ University of Bremen, Germany
}

\begin{abstract}
Fuel mass is one of the main economical and technical restrictions while designing space propulsion systems. Given the high costs related to the transport of mass into space, the necessary fuel mass for accomplishment of the mission should be minimised. For an optimum "thrust/fuel consumption ratio" the gas exit velocity must be maximised. In this research this is achieved through the heating of the micro gas flow by an electrical arc inside the sub-sonic region of the propulsion system. The electrical arc induces a partial ionisation of the propellant gas. Because of the very low mass flow, the gap of the plasma channel has a width of just a few hundred microns. The electrical arc consists of electrons being accelerated through this small gap by the charged walls of the microchannel. The electrons move in a cross flow compared to the propellant gas. The computational results are validated with the experimental data. Through this investigation a very efficient form of electrodynamic heating-modelling is developed. The very good results show the quality of the present method and encourage further utilisation and development. For this reason this model will be used for the optimisation and the computational engineering pre-development of future thermo-electric propulsion systems.
\end{abstract}

Keywords: charge carrier transport, electron flux modelling, electric discharge, charged/neutral particle interaction, electric conductivity. 


\section{Introduction}

The procedure for ionisation of dilute noble gas flows is shown in Figure 1. The accelerated medium is Argon (Ar). The not ionised gas is guided through a ringshaped nozzle. In the narrow neck region an electric arc is used for ionising and heating the gas. Hence the gas exit temperature $T_{2}$ is much higher than the inlet temperature $T_{1}$. In order to study this approach an experimental rig is built up inside a vacuum chamber. The relation between electrical power and mechanical pressurisation is investigated experimentally. The won data are compared with computational results of the electrodynamic behavior inside the micro gap.

The computational model consists of the coupling of the micro gas flow in the trans-sonic thruster application (e.g. [1]) with the heating mechanism of the electron motion including the partial ionisation of the subsonic flow of the electric propulsion system.

\section{Electron transport in ring-shaped gap}

The electron motion inside the ring-shaped volume (Fig. 2) is computed in two different ways. The first approach is based on a 2D channel geometry. Here, the co-axial gap between cathode and anode is modelled by two small 2D channels (Fig. 3). From this point on this approach will be referred to as "channel" geometry. The second approach is defined as "wedge" geometry. The wedge geometry incorporates the spacial expansion of the discretisation volumes in the radial direction.

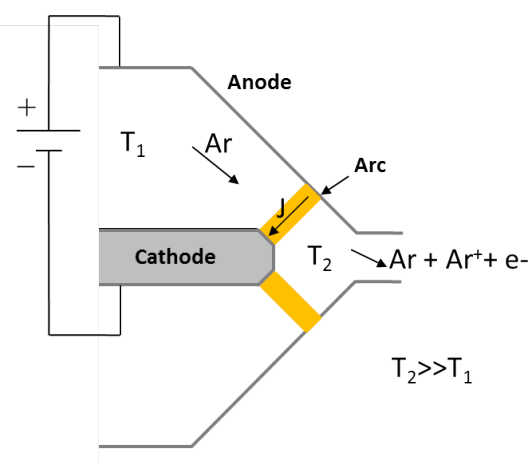

Figure 1: Heating an ionisation in electric arcs.

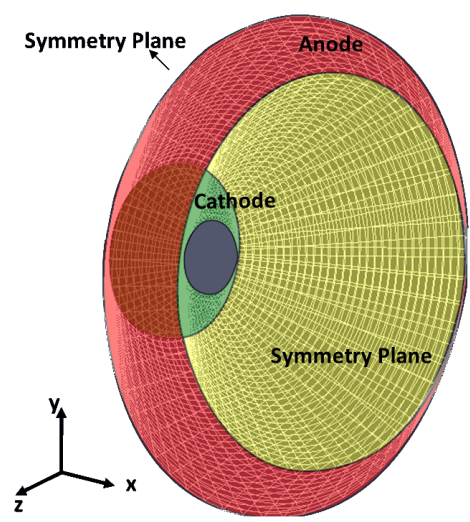

Figure 2: Ionisation volume. 

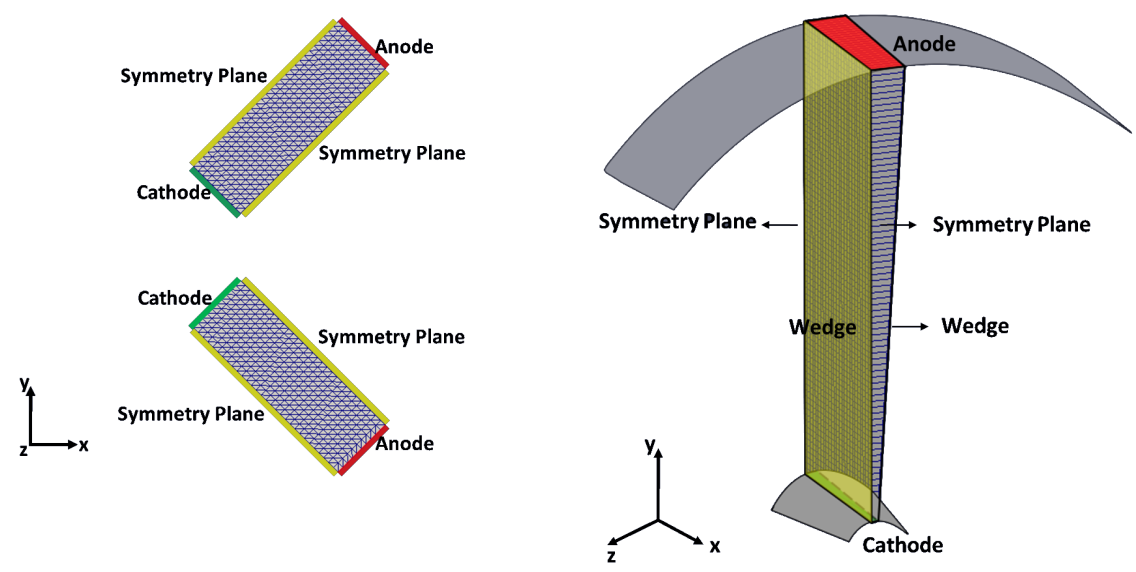

Figure 3: Discretised ionisation gaps. $\quad$ Figure 4: Rotation-symmetric gap.

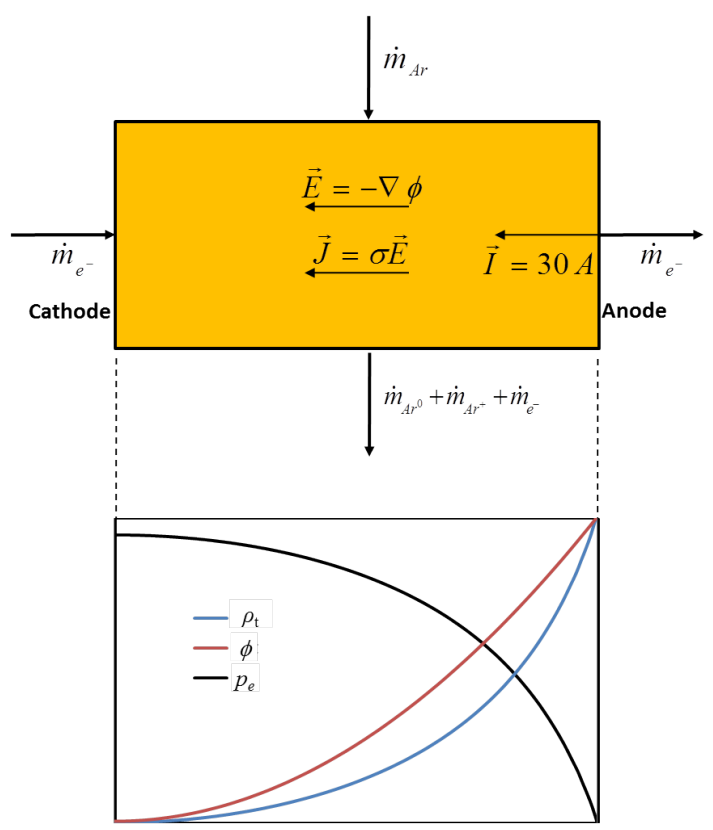

Figure 5: Transport property distribution in ionisation volume.

\subsection{Channel geometry}

In the modelled channels, electrons are accelerated from the cathode to the anode (Fig. 5). The electric field intensity $\vec{E}$ is defined as the negative gradient of the 
electric potential $\phi$. The most essential relation for the process is, however, the definition of the electric conductivity $\sigma$, which describes the relation between the electric flux density $\vec{J}$ and the electric field intensity $\vec{E}$. Furthermore, the flux density is defined as the square-face specific electric current. In the studied case an electrical current of $I=30 \mathrm{~A}$ was used. The electric current is modelled as the motion of charge carriers. The total charge carrier density $\rho_{t}$ is described by the relation (e.g. [2, 3]):

$$
\frac{\rho_{t}}{\epsilon_{0}}=\nabla \cdot \vec{E}=-\Delta \phi
$$

The total charge carrier density $\rho_{t}$ corresponds to the sum of electron charge density $\rho_{e}<0$ and ion charge density $\rho_{i}>0$ :

$$
\rho_{t}=\rho_{e}+\rho_{i} .
$$

A dimensionless imbalance coefficient $0<\eta<1$, which describes the relation between $\rho_{e}$ and $\rho_{i}$, can be defined as follows:

$$
\eta=\frac{\rho_{e}+\rho_{i}}{\rho_{e}} \Rightarrow \rho_{i}=-(1-\eta) \rho_{e}
$$

From the definition of the imbalance coefficient, the following equivalence can be established:

$$
\rho_{t}=\rho_{e}+\rho_{i}=\rho_{e}-(1-\eta) \rho_{e}=\eta \rho_{e} .
$$

Therefore, a negative total charge carrier density is the result of a positive imbalance coefficient $(\eta>0)$. Furthermore, a negative value for $\rho_{t}$ can be

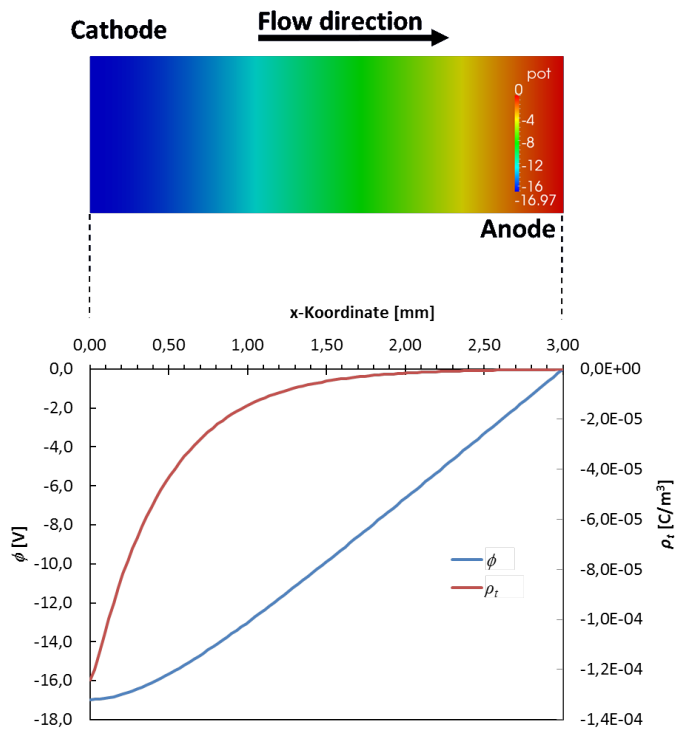

Figure 6: Potential increase in acceleration field. 
explained though the higher electron number density in the ionisation gap due to the presence of the electric arc. With a kinetic approach for the electron motion [4] the (negative) total charge carrier density increases (its absolute value decreases) from the cathode towards the anode (Fig. 6). This is explained by the decreasing number density of (negative charged) electrons towards the anode. The potential increase over the gap depends on the negative cathode voltage and neutral charged anode.

\subsection{Transport equations}

The mathematical model for the electric arc is based on the conservation equations of mass, momentum and energy. The mass density for the charge carriers can be defined as follows:

$$
\rho^{*}=\frac{m_{e}}{e} \rho_{e}
$$

The mass conservation of the charge carriers can be described through the following continuity equation:

$$
\frac{\partial \rho^{*}}{\partial t}+\frac{\partial}{\partial x_{j}}\left(\rho^{*} u_{e j}\right)=0
$$

and from the momentum conservation of the charged carriers following equation results:

$$
\frac{\partial}{\partial t}\left(\rho^{*} u_{e i}\right)+\frac{\partial}{\partial x_{j}}\left(\rho^{*} u_{e i} u_{e j}\right)=-\frac{3}{2} \frac{\partial p_{e}}{\partial x_{j}}-\frac{1}{\sigma}\left(\rho^{*} \frac{e}{m_{e}}\right)^{2} u_{e i} .
$$

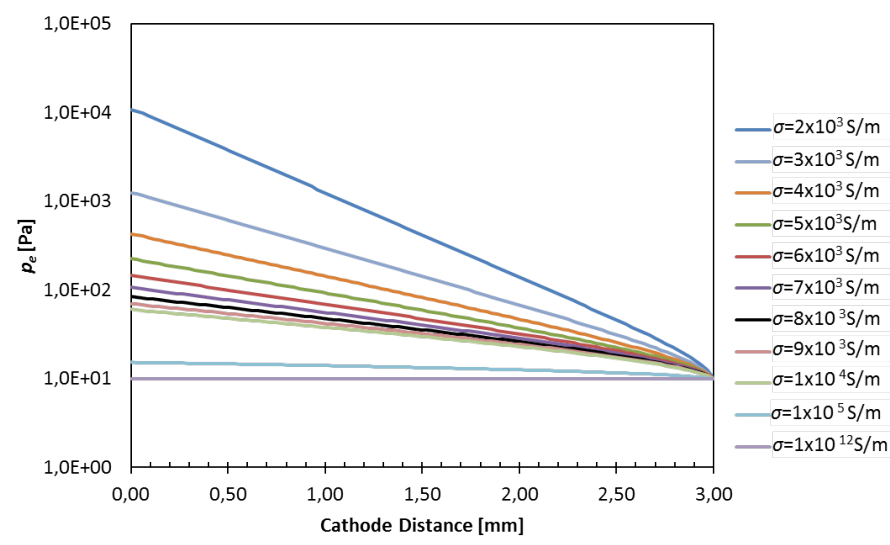

Figure 7: Conduction dependent electron pressure. 


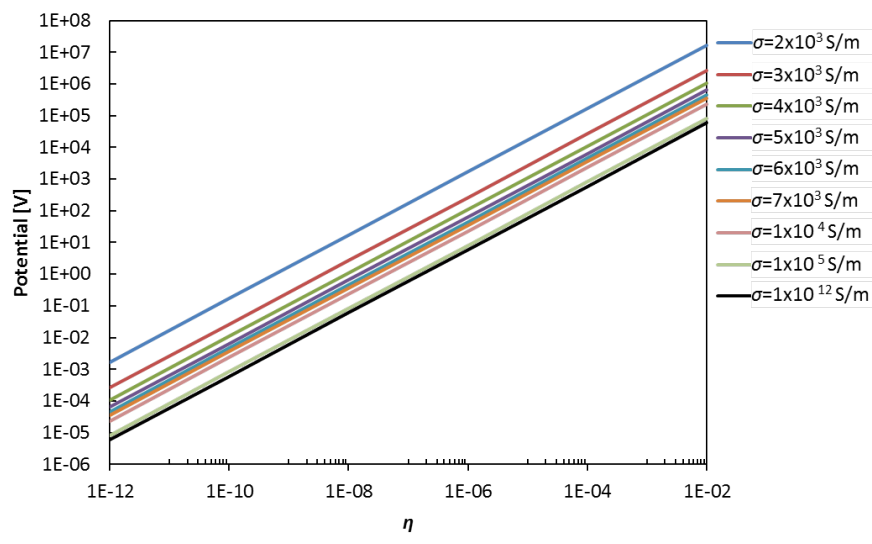

Figure 8: Conduction dependant electric potential.

With the definition of the electron pressure (e.g. [5]):

$$
p_{e}=k_{B} n_{e} T_{e}=\frac{k_{B}}{m_{e}} \rho^{*} T_{e} \Rightarrow \rho^{*}=\frac{m_{e} p_{e}}{k_{B} T_{e}}
$$

the electric conductivity $\sigma$ and the imbalance coefficient $\eta$. The resulting relation between electric conductivity and electron pressure for the channel geometry is shown in Figure 7. Here, the electron pressure at the cathode increases with a decreasing conductivity down to $2 \mathrm{kS} / \mathrm{m}$. The electron pressure at the anode is fixed at $10 \mathrm{~Pa}$ representing the anode entrance work of the charge carriers at the anode surface.

The linear relation between the voltage at the cathode and the imbalance coefficient is shown in Figure 8 for different conductivity values.

\subsection{Wedge geometry}

In order to better represent the rotation-symmetric thruster geometry, a wedge approach is used instead of a 2D flat channel (Fig. 4). With this approach the cell volume increases with the distance from the rotation axis (Fig. 9). Furthermore, the set of transport equations for the modelling of the electric arc is left unchanged (Eq. 1 to 8 ).

The comparison of the results for electron pressure in the wedge geometry in (Fig. 10) with the results in the channel flow computation (Fig. 7) shows the expected extreme non-linear behavior of the electron pressure decrease from the cathode towards the anode surface.

As with the channel approach, the potential drop between cathode and anode on the wedge geometry is linear-dependent from the imbalance coefficient $\eta$ (Fig. 11). However, the conductivity dependence of the voltage drop is much lower on the wedge (Fig. 11) than on the channel geometry (Fig. 8). 


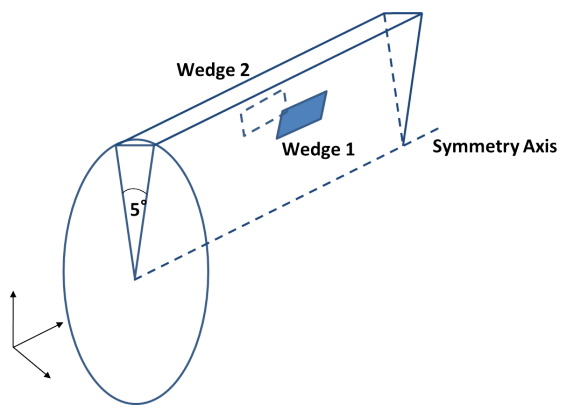

Figure 9: Wedge geometry.

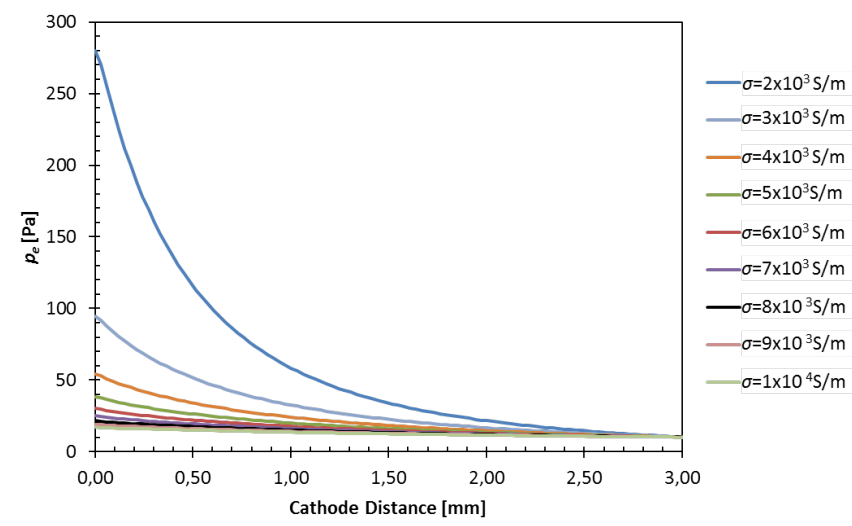

Figure 10: Electron pressure.

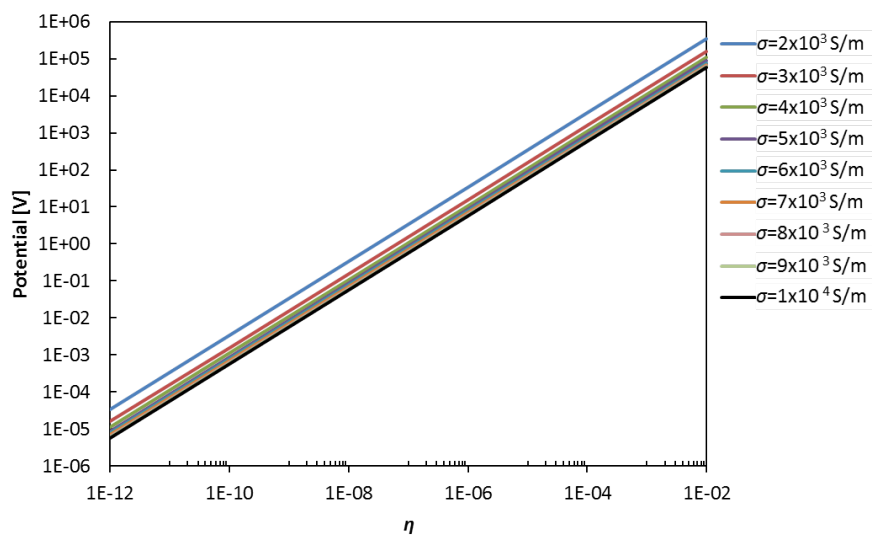

Figure 11: Electric potential. 


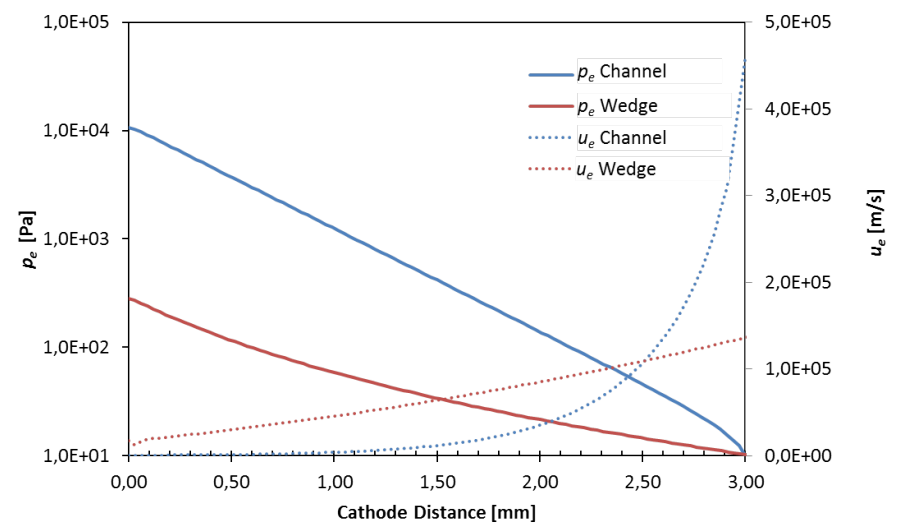

Figure 12: Distribution comparison for wedge and channel geometry.

Figure 12 shows the radial decrease of the electron pressure and the electron acceleration effect for both channel and wedge geometry for an example conductivity of $2 \mathrm{kS} / \mathrm{m}$.

The comparison of two different cathode lengths shows typical differences for electron pressure and velocity. The longer cathode results in a smaller gap between the electrodes (Fig. 13). As a result, the charge carrier density and the electron pressure for the longer cathode are higher compared to the shorter cathode at the same relative anode distance (Fig. 14). As shown in Figure 15, the electron velocity also increases with a longer cathode and a smaller gap between electrodes.

The smaller gap between electrodes results in a higher potential gradient, which leads to higher ionisation degrees, higher temperatures for the micro gas flow

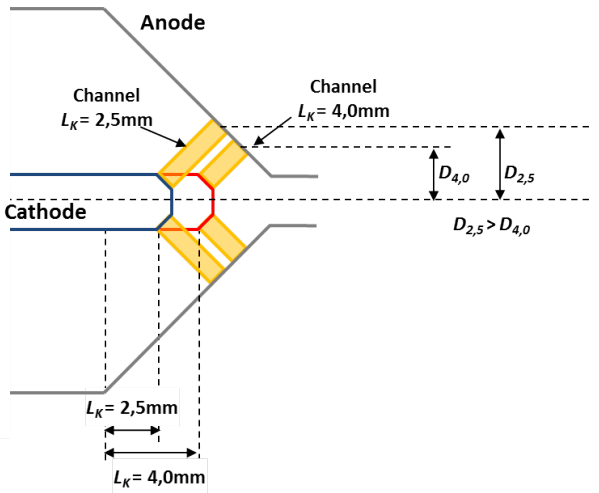

Figure 13: Arc set-up for short and long cathode. 


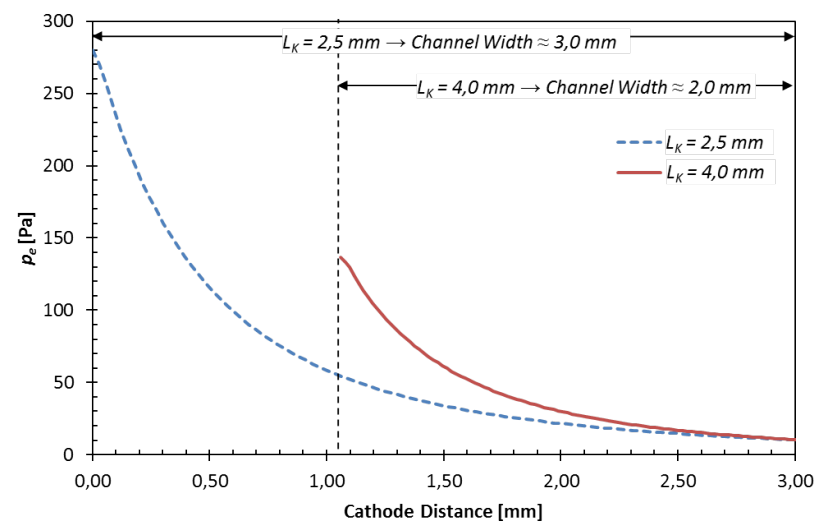

Figure 14: Electron pressure for different gap widths.

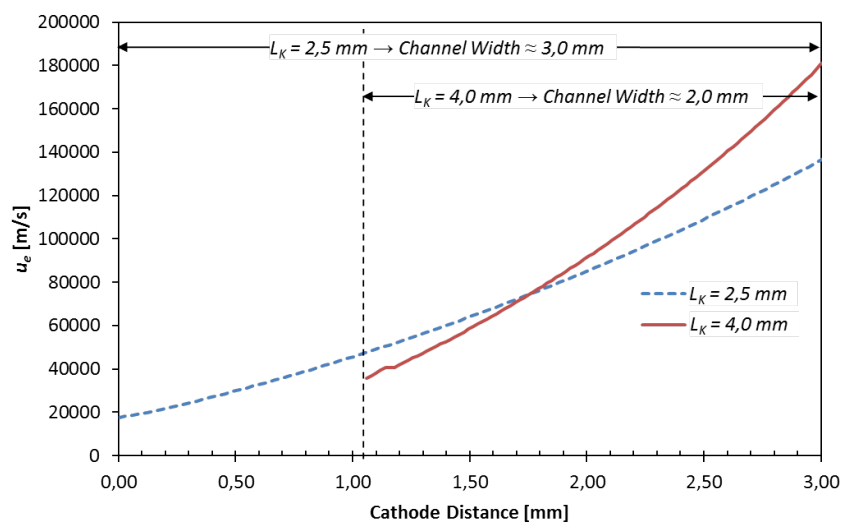

Figure 15: Electron velocity for different gap widths.

(Fig. 16) and marginal higher velocities (Fig. 17). Through experimental results, a mean electrical conductivity of $2 \mathrm{kS} / \mathrm{m}$ for the short cathode, and $10 \mathrm{kS} / \mathrm{m}$ for the long cathode can be established. Therefore, use of the longer cathode leads to an increase of the mean electric conductivity by a factor of 5 .

\section{Thruster simulation}

The configuration with the longer cathode and smaller ionisation gap is investigated numerically with a wedge model for the complete nozzle/diffuser propulsion system. The red line in Figure 18 shows the evaluation path for the 


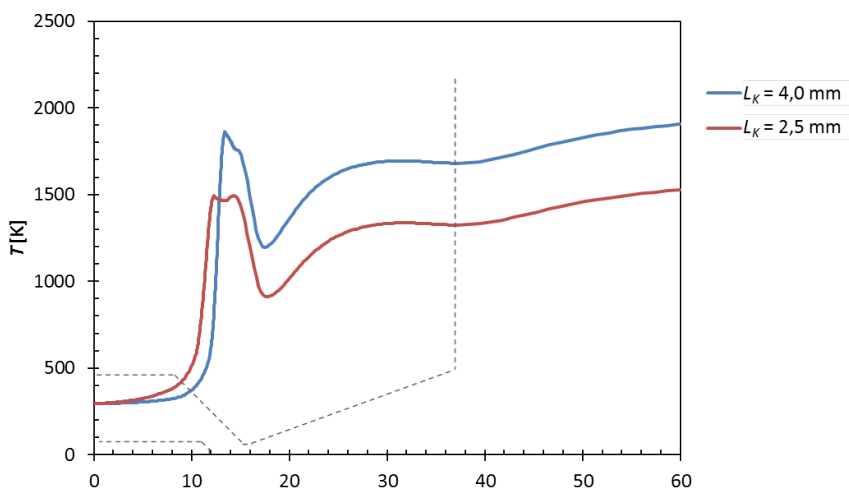

Figure 16: Absolute temperature distribution.

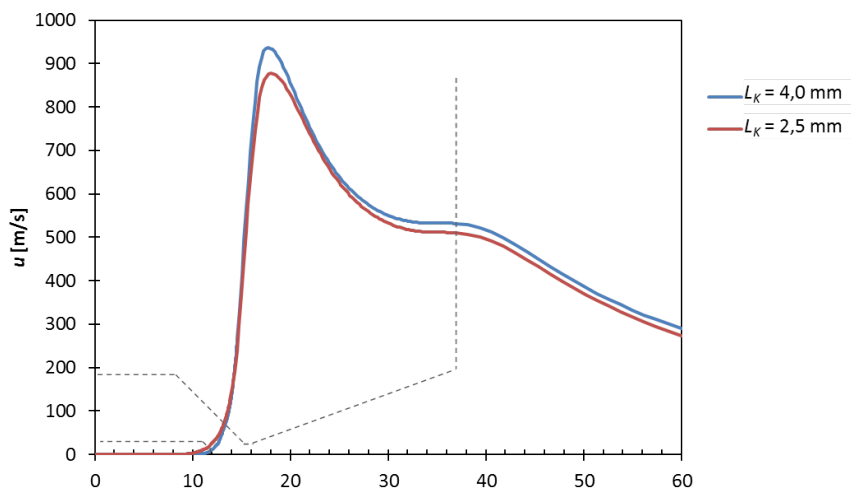

Figure 17: Gas/plasma velocity distribution.

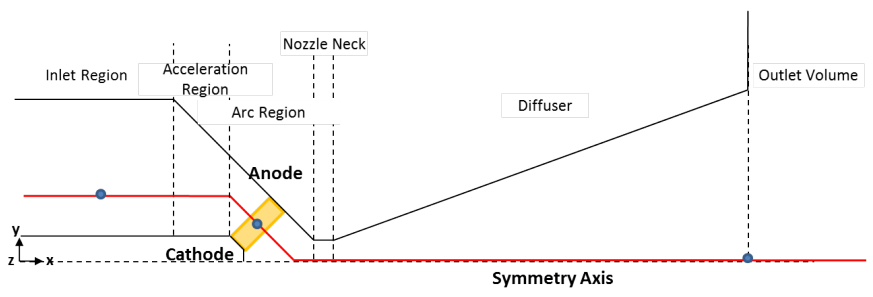

Figure 18: Property distribution path.

transport properties and the yellow area, the arc region where the sub-model for the charge carrier transport is used. 
Three relevant points are investigated: 1) subsonic-region 2) arc-heating 3) diffuser exit. All transport equations and the rotation-symmetric volume are discretised by the finite-volume method (e.g. [6]). The pressure correction is prescribed by a central scheme of Kurganov and Tadmor [7].

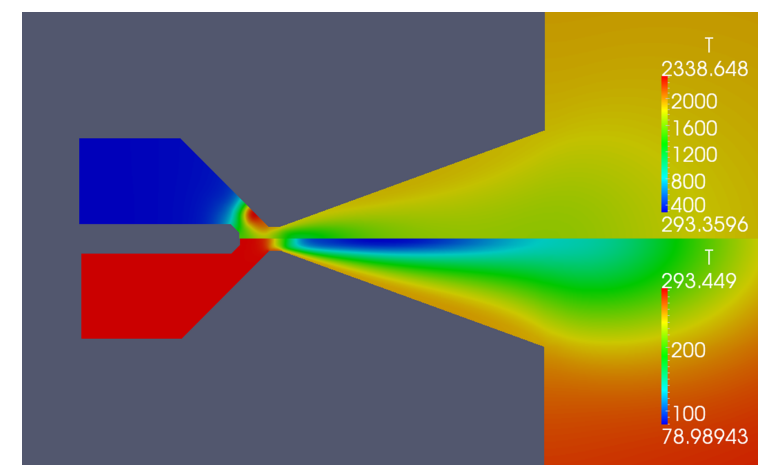

Figure 19: Absolute temperature with (top) and without (bottom) electric arc ionisation.

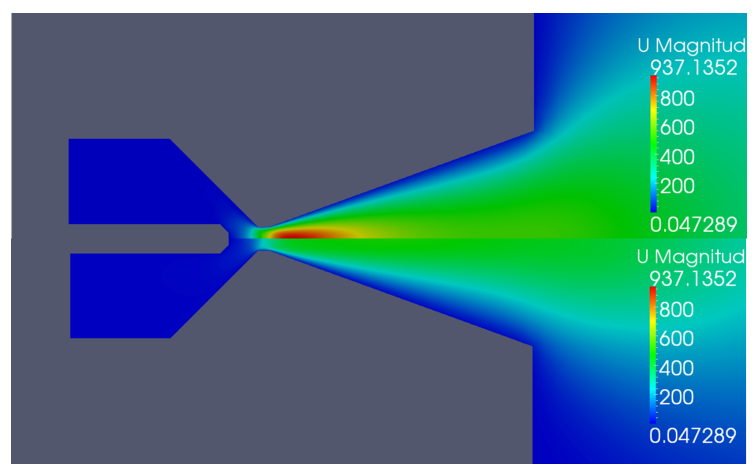

Figure 20: Absolute velocity with (top) and without (bottom) electric arc ionisation.

Based on experimental results the voltage between the electrodes is fixed at $20 \mathrm{~V}$. This allows a better comparison of varying conductivities and electric currents. The temperature difference between the thruster with and without gas heating (ionisation) through electric arc is shown in Figure 19. The legend scale is shifted in order to highlight the temperature ranges of both cold and hot operation of the thruster. The temperature profile along the evaluation path is analysed for different values of electric conductivity. 


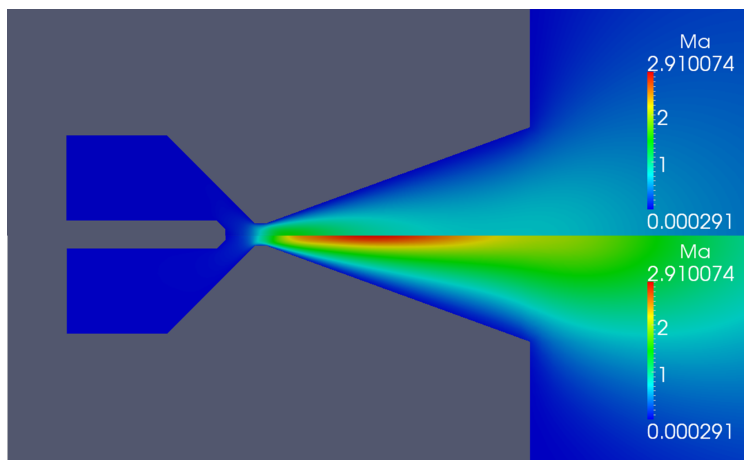

Figure 21: Mach number with (top) and without (bottom) electric arc ionisation.

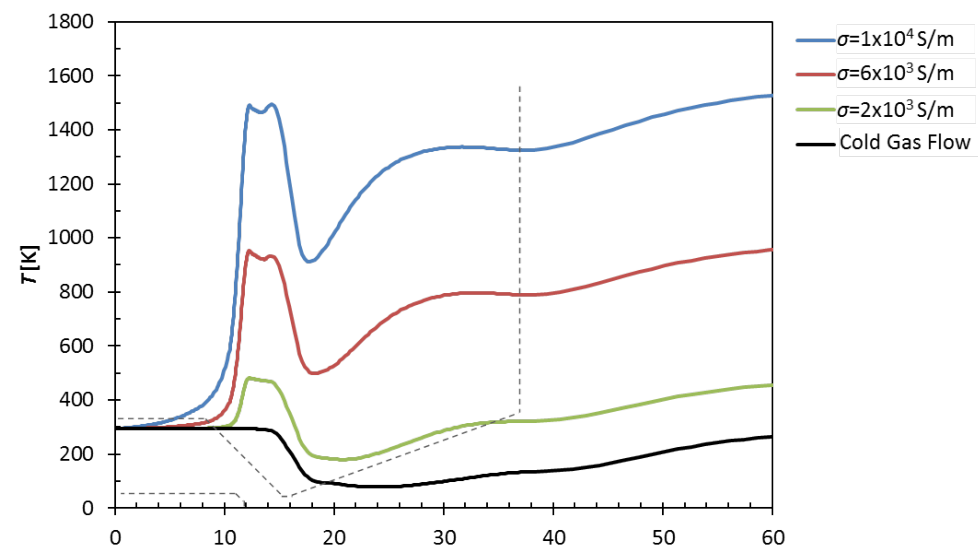

Figure 22: Temperature profile for different electric conductivities and cold operation.

Figure 22 shows a strong temperature increase for higher values for electric conductivity. The same effect results from an increase of the electric current I. The higher temperatures at the nozzle neck increase the local speed of sound $a$. The definition depends on molar mass $M$ and the heat capacity ratio $\gamma$ :

$$
a=\sqrt{\gamma \frac{R_{0}}{M} T}
$$

Moreover, this speed corresponds to the maximum local velocity according to the Laval nozzle principle [8]. Since at the nozzle neck $\mathrm{Ma}=1$ the local flow velocity must increase in order to compensate for the higher local speed of sound. For this 


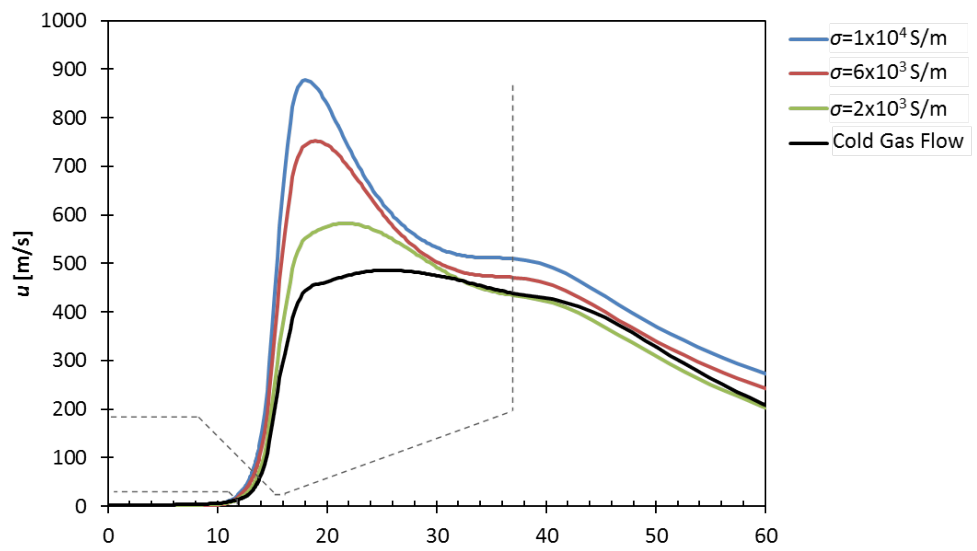

Figure 23: Velocity profile for different electric conductivities and cold operation.

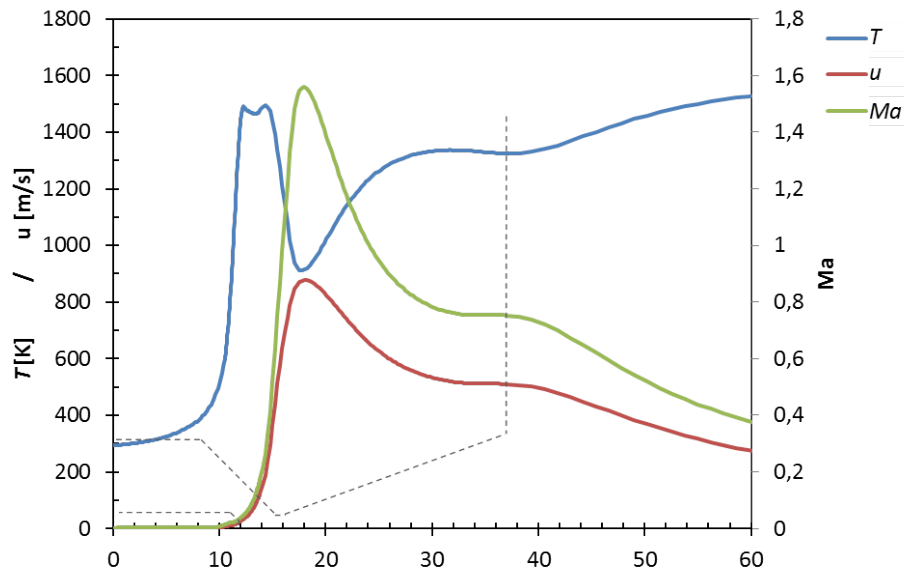

Figure 24: Profiles for different transport values with electric conductivity $10 \mathrm{kS} / \mathrm{m}$.

reason the absolute flow velocities though the nozzle increase with higher values of conductivity and electric current (Figs 20 and 23). With the increasing speed of sound the normalised Mach number

$$
M a=\frac{u}{a}
$$


decreases. The higher local velocities behind the nozzle neck are unable to compensate this tendency (Fig. 21). Figure 24 shows the profiles for velocity $u$, temperature $T$ and Mach number $M a$ along the path shown in Figure 18.

\section{Conclusions}

This work presents a method for the numerical modelling of a micro arc-jet thruster. To achieve this, a sub-model for the electric arc and a model for the transsonic flow, which is partially ionised and heated by the electric arc, are coupled. The results are presented for 2-dimensional and rotational symmetric geometries.

The presented test case of a thermo-electric Argon arc-jet thruster is investigated in order to model the charge imbalance and the electric conductivity inside a laboratory scaled arc-jet with exit flow into a vacuum chamber. The test-case is validated with experimental data. Numerical results are obtained by using the developed model on a rotation symmetric test case. Following facts could be shown by the present coupled model:

- correlation between plasma charge, electron pressure, electric conductivity and imbalance coefficient;

- dependency of electrode gap width and heating effect;

- relation between exit velocity/specific momentum of the electric thruster and electric conductivity.

With the developed coupled-model much better predictions of flow properties and electric requirements are possible, which helps optimise the design and predevelopment phases of micro-electric propulsion systems.

\section{References}

[1] Louwerse, M., Jansen, H., Groenendijk, M. \& Elwenspoek, M., Nozzle fabrication for micro propulsion of a micro satellite. Journal of Microelectromechanical Systems, 19, pp. 61-70, 2009.

[2] Bittencourt, J., Fundamentals of Plasma Physics. Springer: New York, 2004.

[3] Fleisch, D., A Student's Guide to Maxwell Equations. Cambridge, 2008.

[4] Gu, X.J., Barber, R.W. \& Emerson, D.R., How far can 13 moments go in modelling microscale gas phenomena. Thermophysical Engineering, 11, pp. 85-97, 2007.

[5] Haenel, D., Molekulare Gasdynamik: Einführung in die kinetische Theorie der Gase und Lattice-Boltzmann-Methoden. Springer, 2004.

[6] Ferziger, J.H. \& Peric, M., Numerische Strömungsmechanik. Springer: Berlin, 2008.

[7] Kurganov, A. \& Tadmor, E., New high-resolution central schemes for nonlinear conservation laws and convection-diffusion equations. Journal of Compuatational Physics, 160, pp. 241-282, 2000.

[8] Anderson, J., Fundamentals of Aerodynamics. McGraw-Hill: New York, 2001. 